\title{
Erratum to: Gene expression profiling of breast tumor cell lines to predict for therapeutic response to microtubule-stabilizing agents
}

\author{
Gais Kadra $\cdot$ Pascal Finetti $\cdot$ Yves Toiron . \\ Patrice Viens · Daniel Birnbaum · Jean-Paul Borg • \\ François Bertucci • Anthony Gonçalves
}

Published online: 14 February 2012

(C) Springer Science+Business Media, LLC. 2012

\section{Erratum to: Breast Cancer Res Treat \\ DOI 10.1007/s10549-011-1687-8}

Author would like to amend the following corrections in the below Tables 3 and 4 which has been published unnoticed in the original version. Where, the article included an unfortunate inversion between "Neg" and "Pos" rows for "ER", "PR", "Ki67" and "P53" in Tables 3 and 4. In Table 4 , there was also an error in the percentages of grade 1 and 2 tumors with predicted taxane-sensitive and ixabepilone-resistant phenotype. The correct values are given in the tables presented below.

The online version of the original article can be found under doi: 10.1007/s10549-011-1687-8.

G. Kadra · Y. Toiron · J.-P. Borg · A. Gonçalves

Département de Pharmacologie Moléculaire and U891 INSERM,

Centre de Recherche en Cancérologie de Marseille, Institut

Paoli-Calmettes, Marseille, France

P. Finetti - D. Birnbaum - F. Bertucci

Département d'Oncologie Moléculaire and U891 INSERM,

Centre de Recherche en Cancérologie de Marseille, Institut

Paoli-Calmettes, Marseille, France

P. Viens · F. Bertucci · A. Gonçalves ( $\square)$

Département d'Oncologie Médicale and U891 INSERM, Centre

de Recherche en Cancérologie de Marseille, Institut Paoli-

Calmettes, 232 Bd. Ste-Marguerite, 13009 Marseille, France

e-mail: goncalvesa@marseille.fnclcc.fr

P. Viens $\cdot$ J.-P. Borg $\cdot$ F. Bertucci $\cdot$ A. Gonçalves

Université de la Méditerranée, Marseille, France 
Table 3 Clinical, pathological and molecular characteristics of 266 early breast cancer patients, according to their predicted sensitivity to taxanes and ixabepilone

\begin{tabular}{|c|c|c|c|c|c|c|c|}
\hline & $N$ & $\begin{array}{l}\text { Taxane-resistant } \\
N=147\end{array}$ & $\begin{array}{l}\text { Taxane-sensitive } \\
N=119\end{array}$ & $P$-value* & $\begin{array}{l}\text { Ixabepilone-resistant } \\
N=137\end{array}$ & $\begin{array}{l}\text { Ixabepilone-sensitive } \\
N=129\end{array}$ & $P$-value* \\
\hline Age (years) & 266 & & & & & & \\
\hline$\leq 50$ & & $49(33 \%)$ & $53(45 \%)$ & 0.08 & $45(33 \%)$ & $57(44 \%)$ & 0.059 \\
\hline$>50$ & & $98(67 \%)$ & $66(55 \%)$ & & $92(67 \%)$ & $72(56 \%)$ & \\
\hline ER & 264 & & & & & & \\
\hline $\mathrm{Neg}$ & & $23(16 \%)$ & $91(76 \%)$ & $4.71 \mathrm{E}-24$ & $27(20 \%)$ & $87(67 \%)$ & $3.84 \mathrm{E}-15$ \\
\hline Pos & & $122(84 \%)$ & $28(24 \%)$ & & $108(80 \%)$ & $42(33 \%)$ & \\
\hline PR & 264 & & & & & & \\
\hline $\mathrm{Neg}$ & & $35(24 \%)$ & $93(78 \%)$ & $6.48 \mathrm{E}-19$ & $39(29 \%)$ & $89(69 \%)$ & $8.52 \mathrm{E}-11$ \\
\hline Pos & & $110(76 \%)$ & $26(22 \%)$ & & $96(71 \%)$ & $40(31 \%)$ & \\
\hline pT & 245 & & & & & & \\
\hline pT1 & & $45(33 \%)$ & $35(32 \%)$ & 0.89 & $36(29 \%)$ & $44(36 \%)$ & 0.28 \\
\hline pT2-T3 & & $91(67 \%)$ & $74(68 \%)$ & & $88(71 \%)$ & $77(64 \%)$ & \\
\hline Grade & 259 & & & & & & \\
\hline 1 & & $33(23 \%)$ & $12(10 \%)$ & $2.00 \mathrm{E}-05$ & $28(21 \%)$ & $17(13 \%)$ & $3.04 \mathrm{E}-03$ \\
\hline 2 & & $61(43 \%)$ & $28(24 \%)$ & & $54(41 \%)$ & $35(28 \%)$ & \\
\hline 3 & & $48(34 \%)$ & $77(66 \%)$ & & $50(38 \%)$ & $75(59 \%)$ & \\
\hline Ki67 & 227 & & & & & & \\
\hline $\mathrm{Neg}$ & & $55(43 \%)$ & $18(18 \%)$ & $5.44 \mathrm{E}-05$ & $44(38 \%)$ & $29(26 \%)$ & 0.07 \\
\hline Pos & & $72(57 \%)$ & $82(82 \%)$ & & $72(62 \%)$ & $82(74 \%)$ & \\
\hline P53 & 194 & & & & & & \\
\hline $\mathrm{Neg}$ & & $84(76 \%)$ & $41(49 \%)$ & $8.38 \mathrm{E}-05$ & $75(72 \%)$ & $50(56 \%)$ & $2.38 \mathrm{E}-02$ \\
\hline Pos & & $26(24 \%)$ & $43(51 \%)$ & & $29(28 \%)$ & $40(44 \%)$ & \\
\hline Molecular subtype & 266 & & & & & & \\
\hline Basal & & $3(2 \%)$ & $72(61 \%)$ & $1.00 \mathrm{E}-05$ & $11(8 \%)$ & $64(50 \%)$ & $1.00 \mathrm{E}-05$ \\
\hline HER2 & & $12(8 \%)$ & $12(10 \%)$ & & $11(8 \%)$ & $13(10 \%)$ & \\
\hline Luminal A & & $78(53 \%)$ & $11(9 \%)$ & & $65(47 \%)$ & $24(19 \%)$ & \\
\hline Luminal B & & $46(31 \%)$ & $3(3 \%)$ & & $41(30 \%)$ & $8(6 \%)$ & \\
\hline Normal & & $8(5 \%)$ & $21(18 \%)$ & & $9(7 \%)$ & $20(16 \%)$ & \\
\hline Ixabepilone-sensitivity & 266 & & & & & & \\
\hline $\mathrm{Re}$ & & $119(81 \%)$ & $18(15 \%)$ & $2.17 \mathrm{E}-28$ & - & - & \\
\hline $\mathrm{Se}$ & & $28(19 \%)$ & $101(85 \%)$ & & - & - & \\
\hline
\end{tabular}

* Fisher's exact test 
Table 4 Clinical, pathological and molecular characteristics of 46 early breast cancer patients, predicted to have differential sensitivity to taxanes and ixabepilone

* Fisher's exact test

\begin{tabular}{|c|c|c|c|c|}
\hline & $N$ & $\begin{array}{l}\text { Ixabepilone-sensitive } \\
\text { Taxane-resistant } \\
N=28\end{array}$ & $\begin{array}{l}\text { Taxane-sensitive } \\
\text { Ixabepilone-resistant } \\
N=18\end{array}$ & $P$-value* \\
\hline Age (years) & 46 & & & \\
\hline$\leq 50$ & & $9(32 \%)$ & $5(28 \%)$ & 1 \\
\hline$>50$ & & $19(68 \%)$ & $13(72 \%)$ & \\
\hline ER & 46 & & & \\
\hline $\mathrm{Neg}$ & & $10(36 \%)$ & $14(78 \%)$ & $7.20 \mathrm{E}-3$ \\
\hline Pos & & $18(64 \%)$ & $4(22 \%)$ & \\
\hline PR & 46 & & & \\
\hline $\mathrm{Neg}$ & & $10(36 \%)$ & $14(78 \%)$ & $7.20 \mathrm{E}-3$ \\
\hline Pos & & $18(64 \%)$ & $4(22 \%)$ & \\
\hline $\mathrm{pT}$ & 42 & & & \\
\hline pT1 & & $13(48 \%)$ & $4(27 \%)$ & 0.21 \\
\hline pT2-T3 & & $14(52 \%)$ & $11(73 \%)$ & \\
\hline Grade & 46 & & & \\
\hline 1 & & $8(29 \%)$ & $3(17 \%)$ & 0.14 \\
\hline 2 & & $10(36 \%)$ & $3(17 \%)$ & \\
\hline 3 & & $10(36 \%)$ & $12(66 \%)$ & \\
\hline Ki67 & 39 & & & \\
\hline Neg & & $14(56 \%)$ & $3(21 \%)$ & 0.049 \\
\hline Pos & & $11(44 \%)$ & $11(79 \%)$ & \\
\hline P53 & 34 & & & \\
\hline $\mathrm{Neg}$ & & $17(85 \%)$ & $8(57 \%)$ & 0.12 \\
\hline Pos & & $3(15 \%)$ & $6(43 \%)$ & \\
\hline Molecular subtype & 46 & & & \\
\hline Basal & & $1(4 \%)$ & $9(50 \%)$ & $1.50 \mathrm{E}-04$ \\
\hline HER2 & & $5(18 \%)$ & $4(22 \%)$ & \\
\hline Luminal A & & $14(50 \%)$ & $1(6 \%)$ & \\
\hline Luminal B & & $6(21 \%)$ & $1(6 \%)$ & \\
\hline Normal & & $2(7 \%)$ & $3(17 \%)$ & \\
\hline
\end{tabular}

